\title{
BMJ Open Interdisciplinary systematic review: does alignment between system and design shape adoption and use of barcode medication administration technology?
}

\author{
Rachel Williams (D) , ${ }^{1}$ Reham Aldakhil, ${ }^{2}$ Ann Blandford (iD) , ${ }^{3}$ Yogini Jani (D) ${ }^{1,4}$
}

To cite: Williams $R$,

Aldakhil $\mathrm{R}$, Blandford $\mathrm{A}$, et al. Interdisciplinary systematic review: does alignment between system and design shape adoption and use of barcode medication administration technology? BMJ Open 2021;11:e044419. doi:10.1136/ bmjopen-2020-044419

- Prepublication history and additional supplemental material for this paper are available online. To view these files, please visit the journal online (http://dx.doi.org/10.1136/ bmjopen-2020-044419).

Received 03 September 2020 Accepted 09 June 2021

Check for updates

(c) Author(s) (or their employer(s)) 2021. Re-use permitted under CC BY-NC. No commercial re-use. See rights and permissions. Published by BMJ.

${ }^{1}$ Centre for Medicines Optimisation Research and Education, University College London NHS Foundation Trust, London, UK

${ }^{2}$ Clinical and Research Informatics Unit, UCL Institution of Health informatics, University College London, London, UK ${ }^{3} \mathrm{UCL}$ Institute of Healthcare Engineering, University College London, London, UK

${ }^{4}$ UCL School of Pharmacy, University College London, London, UK

Correspondence to Dr Yogini Jani; y.jani@ucl.ac.uk

\section{ABSTRACT}

Background In order to reduce safety risks associated with medication administrations, technologies such as barcode medication administration (BCMA) are increasingly used. Examining how human factors influence adoption and usability of this technology can potentially highlight areas for improvement in design and implementation.

Objective To describe how human factors related determinants for BCMA have been researched and reported by healthcare and human-computer interaction disciplines.

Data sources The Cumulative Index of Nursing, and Allied Health Literature, PubMed, OVID MEDLINE and Google Scholar.

Study eligibility criteria Primary research published from April 2000 to April 2020, search terms developed to identity different disciplinary research perspectives that examined BCMA use, used a human factors lens and were published in English.

Synthesis methods Computerised systematic searches were conducted in four databases. Eligible papers were systematically analysed for themes. Themes were discussed with a second reviewer and supervisors to ensure they were representative of content.

Results 0 f 3707 papers screened, 11 were included. Studies did not fit neatly into a clinical or human-computer interaction perspective but instead uncovered a range of overlapping narratives, demonstrating consensus on the key themes despite differing research approaches. Prevalent themes were misaligned design and workflow, adaptation and workarounds, mediating factors, safety, users' perceptions and design and usability. Inadequate design frequently led to workarounds, which jeopardised safety. Reported mediating factors included clarity of user needs, pre/post implementation evaluations, analysis of existing workarounds and appropriate technology, infrastructure and staffing.

Limitations Most studies were relatively small and qualitative, making it difficult to generalise findings. Conclusion Evaluating interdisciplinary perspectives including human factors approaches identified similar and complementary enablers and barriers to successful technology use. Often, mediating factors were developed to compensate for unsuitable design; a collaborative
Strengths and limitations of this study

- The search strategy captured literature from both healthcare and human-computer interaction perspectives, providing a rich understanding of the factors.

- A second reviewer repeated the initial search with a high level of agreement and reviewed the data extraction process and theme selection to ensure findings were representative.

- The Preferred Reporting Items for Systematic Reviews and Meta-Analyses checklist was used to design the study protocol.

- Most studies included were relatively small in terms of number of participants and were usually conducted in just one or two hospitals, primarily in the USA.

- Qualitative methodology was prevalent in the selected studies, making it difficult to generalise findings.

approach between system designer and end users is necessary for BCMA to achieve its true safety potential.

\section{BACKGROUND}

The prevalence and subsequent harm caused by medication errors has galvanised efforts to develop systems, policies and technologies to prevent them. ${ }^{1-5}$ Medication administration errors are the most common adverse events in hospitals; it has been estimated that a patient will experience one medication error per 24 hours as an inpatient. ${ }^{6} 7$ Annually, an estimated 237 million 'medication errors' occur in the NHS in England, $72 \%$ do not cause harm but 66 million are clinically significant. Avoidable adverse drug reactions contribute to an estimated 1700 deaths per year, at a financial cost of $£ 98.5$ million. ${ }^{4}$

Medication management and administration in the hospital setting encompass a complex and interlinked series of events and individuals, including pharmacists, doctors, 
nurses, stock managers and patients. There are many opportunities in this chain to intercept errors which may lead to adverse events, and it is hard to estimate how many potential errors are intercepted before they reach the patient. ${ }^{4}$ However, medication administration has been identified as the phase where interception of a medication error is least likely to occur, with only about $2 \%$ of errors being intercepted at the point of administration. ${ }^{7-10}$ To mitigate some of these risks, barcode medication administration (BCMA), usually in conjunction with an electronic medication administration record (eMAR), has been promoted to reduce the prevalence of medication administration errors. ${ }^{1112}$

Bates argues that the causes of frequent medication error are relatively simple: the bulk of the systems in place were not formally designed and are not subject to the stringent regulation processes used in other high-risk industries such as aviation. ${ }^{13}$ Furthermore, healthcare is complex: it is highly regimented and systematic while also being unpredictable, requiring clinicians to constantly learn alongside their practice, often adapting to conform to local policies; this presents many challenges for clinicians navigating safe practice. ${ }^{14}$ Health information technologies (HITs), such as BCMA, seek to ensure safety for both patient and clinician.

BCMA technology incorporates the 'five rights of medicines administration' (right drug, right time, right patient, right dose, right route) into an automated system. ${ }^{15} 16$ BCMA automates and records each medication administration and prompts the user to ensure it meets the required safety standard, warning the user if any discrepancy between prescription and administration detail is identified. For example, if the barcoded patient identification band does not match the selected electronic medication chart, an alert will notify the user of the mismatch and prompt them to check they have the right medication for the right patient, potentially avoiding a "wrong patient' error. ${ }^{11}$ While BCMA technology can reduce some medication errors by streamlining workflow and improving medicine and patient identification rates ${ }^{17}$ it can exacerbate others or even cause new types of error to occur. $^{11-13}$ The literature presents a complex picture of unintended consequences following BCMA implementation, indicating that the overall effect of a new HIT, such as BCMA, is often difficult to predict. ${ }^{13} 18$

From a human factors perspective, the belief that adopting HITs such as BCMA will lead to improved safety outcomes is termed 'magical thinking'; rather, successful adoption is complex, reliant on many mediating factors and context dependent. ${ }^{19} 20$ The introduction of any new work system will have a transformative effect on the established workflow; successful adoption is not guaranteed, but a positive outcome may result from the comparison and clarification of the established and proposed systems. ${ }^{20-23}$ However, unintended consequences such as workarounds may also occur.

Human factors models such as systems engineering in patient safety (SEIPS) have been instrumental in understanding the factors that influence successful implementation of BCMA and other HIT. ${ }^{24}$ Such models examine the wider context in which work takes place, acknowledging that adverse events are rarely caused by one individual, but from a series in interconnected events. ${ }^{25} \mathrm{~A}$ human factors lens can be used to examine multiple factors such as environment, organisation, technology and tasks, to gain understanding of why errors occur and how to prevent them. ${ }^{25}$

This literature review identifies factors which enable and limit the use of BCMA, during the implementation phase and beyond, by using a human factors lens to capture primary research from both users and implementers of the technology. Human factors approaches can often expose the root causes of undesirable outcomes, and by using a search strategy that captures research from across the spectrum of those designing and using the technology, it may be possible to develop implementation strategies that enable effective BCMA implementation and long-term use.

\section{METHOD}

\section{Search strategy}

Multiple key words were developed using terminology that would identify literature from healthcare, design, and informatics perspectives using a human factors lens. The Preferred Reporting Items for Systematic Reviews and Meta-Analyses (PRISMA) was used as a guide for literature review protocol development. ${ }^{26}$ The Cumulative Index of Nursing, and Allied Health Literature, PubMed, OVID MEDLINE and Google Scholar were systematically searched for literature produced between April 2000 and April 2020. Search terms were combined with Boolean operators and were adapted to match database terms. A document detailing the search strategy is available as a online supplemental file (Search Strategy).

\section{Selection process}

The selection process is displayed in figure 1. Full-text, English language, peer-reviewed papers of primary research were included; grey literature and literature reviews were excluded. The results from each database were compared and duplicates removed. Abstracts of the remaining papers were reviewed against the inclusion criteria and if the study included BCMA, usability and a human factors approach, it was considered eligible and the full text was reviewed for inclusion. The paper did not have to explicitly state human factors in the title, as long as human factors principles were evident in the methodology. For example, workarounds are frequently studied in relation to BCMA; studies using human factors principle to understand the causes of workarounds were included, but studies examining workaround prevalence, in relation to error, without examining underlying causes were excluded.

\section{Data extraction process}

A second reviewer (RA) repeated the search and study selection process, resulting in a high level of agreement 


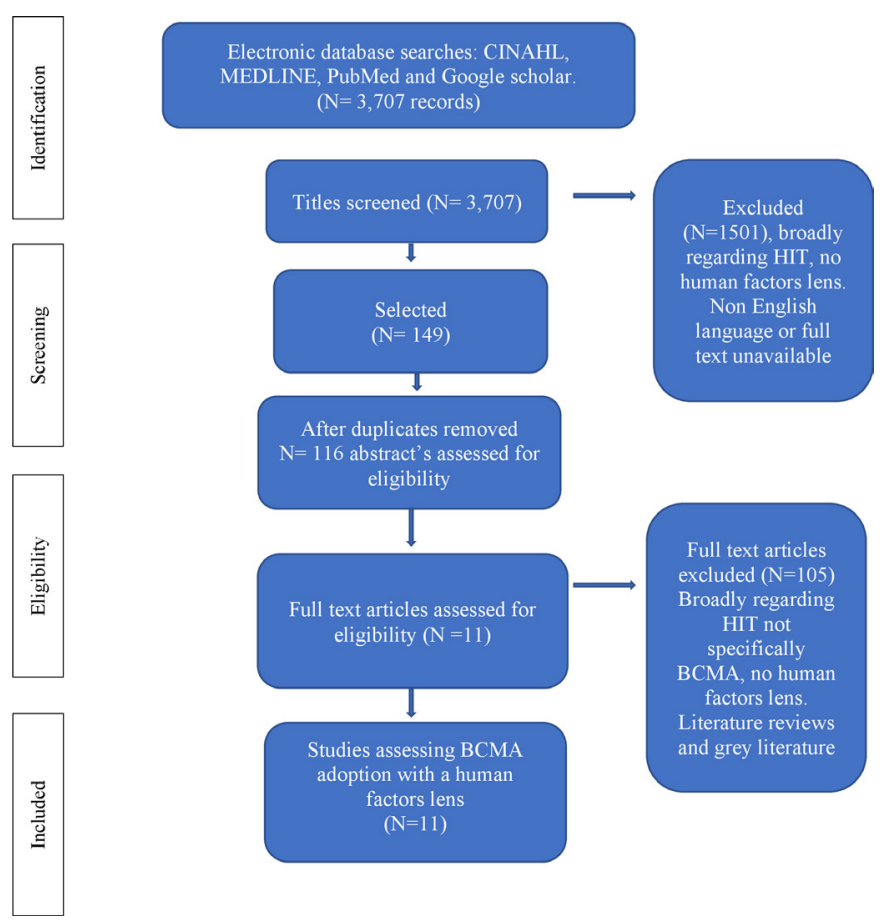

Figure 1 PRISMA flow chart. Detailing selection process of studies reviewed. BCMA, barcode medication administration; CINAHL, Cumulative Index of Nursing, and Allied Health Literature; HIT, health information technology; PRISMA, Preferred Reporting Items for Systematic Reviews and MetaAnalyses.

(76\%) for study eligibility through titles review. The level of agreement for final inclusion was very high, with both reviewers agreeing on 10 of the 11 studies; following discussion all 11 were included in the review. Thematic data extraction was performed by RW, with the emergent themes developed iteratively through discussion with $\mathrm{AB}$ and YJ. RA reviewed a selection of the papers and associated thematic extraction and agreed that the identified themes were appropriate and representative of the study findings.

\section{Patient and public involvement}

No patient or public involvement was sought in the development and execution of the literature review. No personal or identifying private health information would be derived from the public sources being searched.

\section{RESULTS}

\section{Study characteristics}

Overall, 9 of the 11 papers included were primary studies. The exceptions were Novak et als 2013 study, ${ }^{27}$ which reanalysed data from two previous studies ${ }^{28} 29$ (both included in the selected studies) to examine a new research question and Van der Veen $e t a l$ 's 2020 study $^{11}$ on factors which contribute to the occurrence of workarounds, which reanalysed data from their 2018 study $^{30}$ to explore a different facet to the original research (also included in the selected papers).
Various study designs and methodologies were used to investigate BCMA implementation and use. All studies were qualitative or mixed methods, gathering data by observation of practice or a combination of observation, survey, focus groups and interviews. Multiple papers also collected quantitative data, such as medication error reports ${ }^{31}$ and BCMA override data. ${ }^{732}$ Theoretical frameworks were used in all studies except for Van der Veen $e t$ $a l$ s work. ${ }^{1130}$ The majority of the frameworks originated in the human factors field, including SEIPS, the technology acceptance model and complexity theory. Full details of the frameworks used are listed in table 1. Three studies used statistical methods to analyse their findings, Patterson et al established statistical significance of a higher incidence of workarounds in long-term care when compared with acute care $(93 \%$ vs $23 \%, \mathrm{p}<0.001) .{ }^{32}$ Van der Veen et al used logistic regression analysis to assess the association between workarounds and medication error and identify factors which contribute to the occurrence of workarounds. ${ }^{11}{ }^{30}$ Holden et al used regression models to predict acceptance of new technologies, using general linear mixed models with repeated measures to examine user perception of BCMA both pre and post implementation. ${ }^{20}$ Further studies led by Rack $e t a l^{31}$ and Koppel $e t a l^{7}$ presented survey results and override data as percentages of agreement but did not present any further statistical analysis. The remaining studies used thematic analysis to establish emergent themes, with differing methods. Holden $e t a l$ s 2013 study used descriptive coding ${ }^{28}$ and Novak $e t$ l 's 2012 study used qualitative data analysis software to transcribe and analyse fieldnotes, ${ }^{29}$ whereas Novak $e t$ als 2013 study used researchers independently assessing their fieldnotes for themes before discussing as a group and finalising theme inclusion. ${ }^{27}$ Staggers et al $\mathrm{s}$ study $^{33}$ differed from the others in terms of data collection and analysis: this team studied online BCMA training routinely undertaken by nurses. The researchers used heuristic evaluation methods to establish usability problems with the technology and rate how this affected users' situational awareness. A severity score was then assigned to the usability problem to establish the safety risk posed by the usability issue identified. Studies varied in terms of length, number of participants, use of comparison sites, $\mathrm{pre} /$ post analysis and settings as detailed in table 1.

\section{Research focus}

The studies included in this review use human factors methods with a range of research focuses and diverse narratives on BCMA adoption, use and success. Holden $e t$ $a l,{ }^{2834}$ Novak $e t a l^{29}$ and Staggers $e t a l^{33}$ studied the design and usability of BCMA systems and the effects of preexisting workflows at various stages of BCMA implementation and use. The safety risks introduced by poorly aligned BCMA design and clinical workflow were acknowledged as a distal outcome of poor design but were not the focus of these studies. Rather, this group of studies highlights how workarounds can identify design flaws. This is in line with Koppel $e t a l \mathrm{~s}^{7}$ and Rack $e t a l \mathrm{~s}^{31}$ studies on the causes 


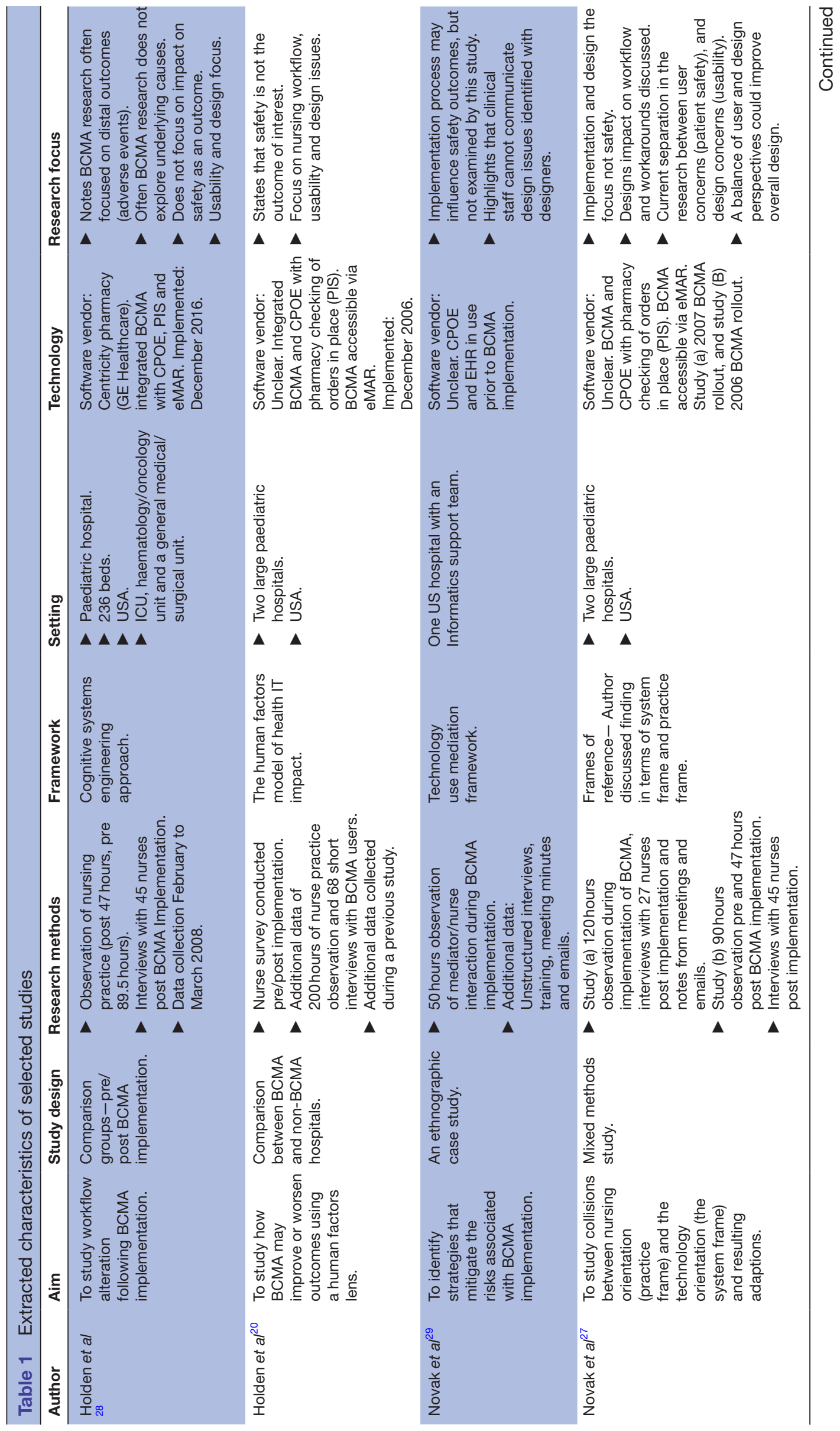




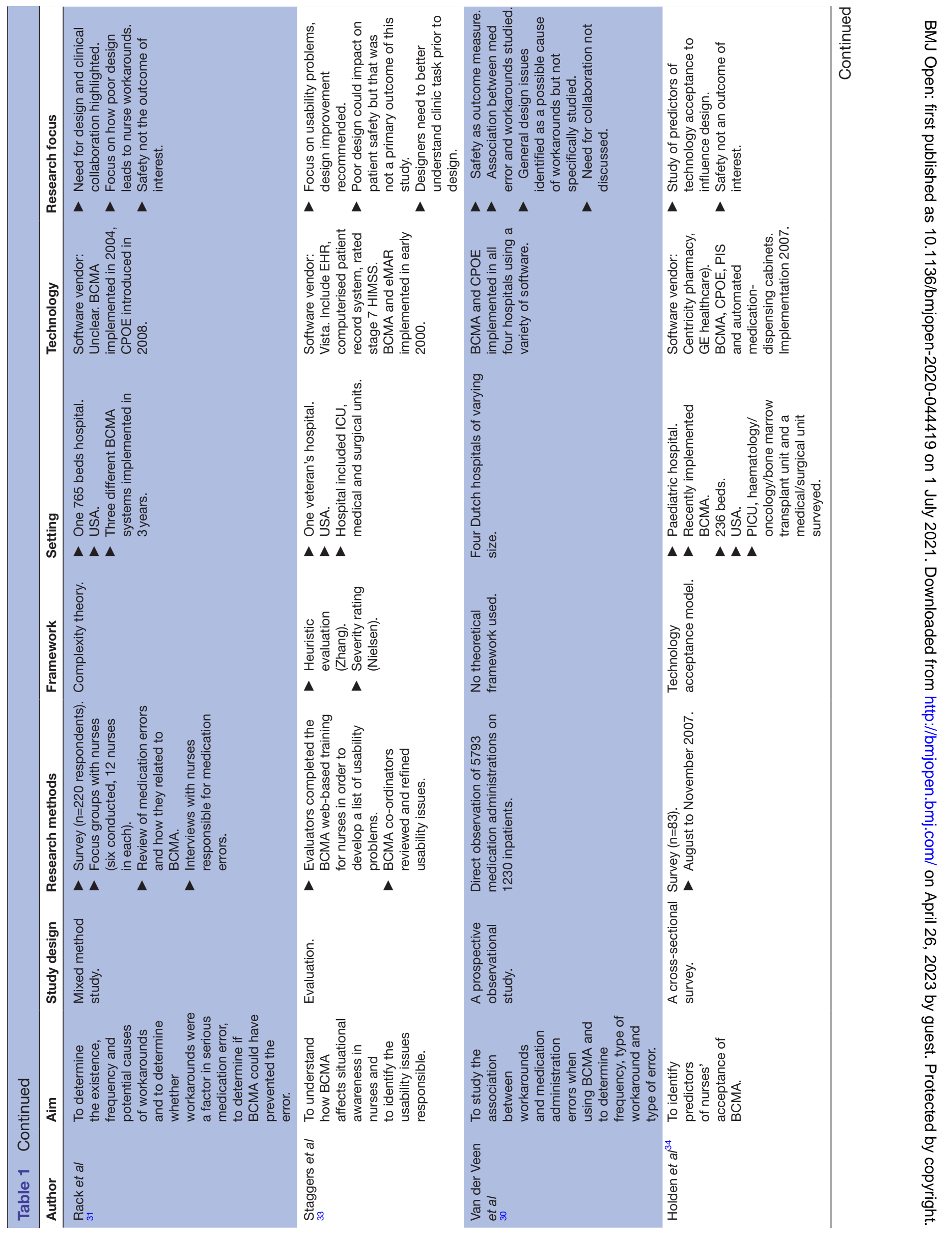




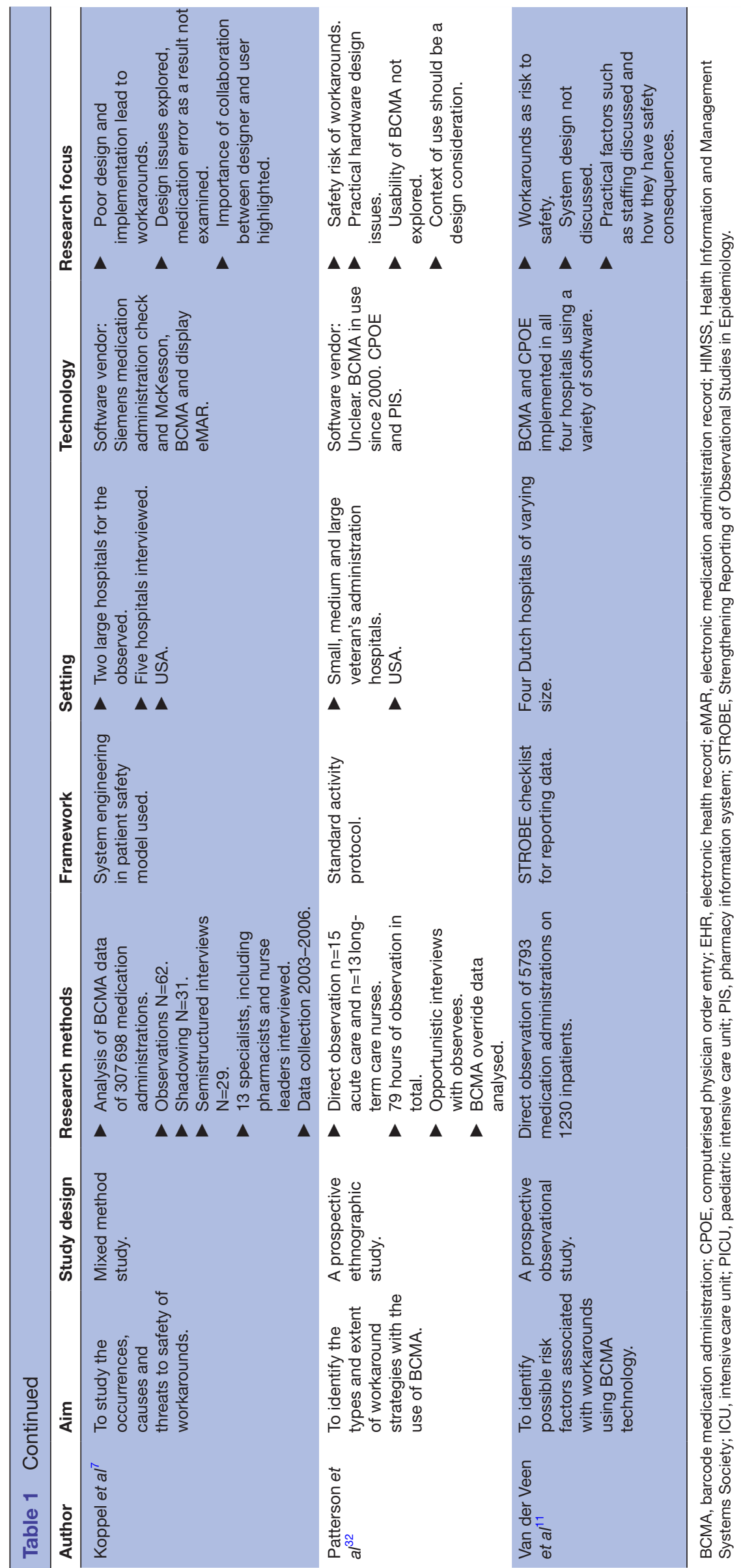


and frequency of workarounds; they concluded that poor design could increase their prevalence and have longterm consequences for safety while not explicitly studying design issues or safety outcomes and instead focusing on workarounds. In parallel, Van der Veen $e t a l^{1130}$ and Patterson $e t a l^{2}$ studied the patient safety risk presented by the use of workarounds in the clinical setting, focusing on the consequences of circumventing the safety features of BCMA, acknowledging that their root may be in poor design, but not further commenting on particular design failures. Holden $e t a l^{34}$ examined users' perspectives of BCMA use pre and post implementation, adding another dimension to understanding technology acceptance and suggesting that user perception and not just the study of workarounds can aid iterative design. A further perspective is presented in Novak et $a l \mathrm{~s}^{27}$ study of an informatics team which implemented BCMA technology into clinical practice; as professionals with both clinical and informatics expertise, their experience is highly valuable to those planning to implement BCMA technology into the healthcare setting.

The differing research focuses in the field of BCMA study is discussed in two of the papers. ${ }^{27}{ }^{28}$ Holden et $a l^{28}$ noted that BCMA research routinely focuses on the relationship between adverse events and workarounds, arguing that investigating the outcome alone does not enable identification of the causes of workarounds and neglects design issues that may be responsible. Novak et $a l^{27}$ propose that future research must do more to understand the perspective of the workers, designers and implementers, to better understand factors affecting successful BCMA use.

\section{THEMES}

Each study employed unique approaches to better understand BCMA use and success; nevertheless, many themes were evident in multiple studies. The main themes identified were misaligned design and workflow, adaptation and workarounds, factors which mediate BCMA use, safety, users' perception and design and usability. A summary of these themes is presented in table 2 .

\section{Misaligned design and workflow}

Many studies found that BCMA system design and clinical workflow were misaligned, limiting the user's ability to plan ahead and prioritise. ${ }^{2027-33}$ This mismatch seemed to result from BCMA design underestimating the complexity of nurses work and how frequently they have to adapt to individual, environmental, institutional and technological factors beyond their control. ${ }^{31}$

During direct observation, nurses were seen to frequently adapt and reorganise their work to achieve their goals and optimise patient care, putting them at odds with the sometimes inflexible BCMA design. ${ }^{27}{ }^{33} \mathrm{~A}$ frequent observation was that BCMA design focuses the user on single timepoints, assuming that nurses complete tasks at scheduled times, whereas in practice nurses' work involves prioritisation, making the importance of timeliness context dependent. ${ }^{20} 273133$ BCMA design attempts to focus the user on the specific task of medication administration, but multiple studies found that nurses could not easily access additional information required to safely administer medication such as vital signs, medical history and information regarding previous or future doses. ${ }^{27} 3133$ Holden et al found that this prescriptive design limited users' critical thinking and therefore posed a safety risk. ${ }^{34}$ Nurses were observed to use paper to record pertinent information because the BCMA design did not give them an overview of their tasks or patients and limited their ability to communicate with colleagues. ${ }^{28}$ Staggers et als study of situational awareness found 99 usability issues with the BCMA system studied, of which 15 were rated catastrophic, arguing that the design did not match the way nurses think or work. ${ }^{33}$ Van der Veen et al also found that the BCMA did not fit well with daily workflow of nurses who encountered both software and hardware blockades. ${ }^{11}$

\section{Adaptations and workarounds}

All studies which conducted observation in the clinical setting reported workarounds associated with BCMA technology. Although the consequences and causes of workarounds varied greatly, there was agreement that workarounds undermined the safety features of BCMA technology.

Patterson et als BCMA compliance study found that workarounds reduced technology effectiveness and increased the risk of adverse events. ${ }^{32}$ Van der Veen et al found a statistically significant relationship between workarounds and medication error: $6 \%$ of the workarounds resulted in the wrong dose being administered and $78 \%$ of the workarounds were medication omissions. ${ }^{30}$ Van der Veen et al reanalysed these data to look for factors which made workarounds more likely, finding a statistically significant relationship between high patient to nurse ratios and workarounds, arguing that increased work pressures led to an increase in the prevalence of workarounds. ${ }^{11}$

Holden et al found that BCMA triggered multiple types of problem-solving behaviours. They noted that the problem solving itself was a 'double edged sword', preventing failures missed in the design process, thus concealing design flaws, preventing redesign. ${ }^{28}$ For example, the use of paper artefacts to record patient information is potentially dangerous because it is not available to the wider clinical team and the shared information may be out of date. The use of paper artefacts conceals the user need and introduces a safety risk, which could be alleviated by better design.

Using the SEIPS framework to examine technological, task, organisational, patient related or environmental causes of workarounds, Koppel et al found that workarounds were complex, resulting from numerous causes and themselves creating additional workarounds. ${ }^{7}$ Koppel $e t$ al and Holden et al suggest that workarounds 


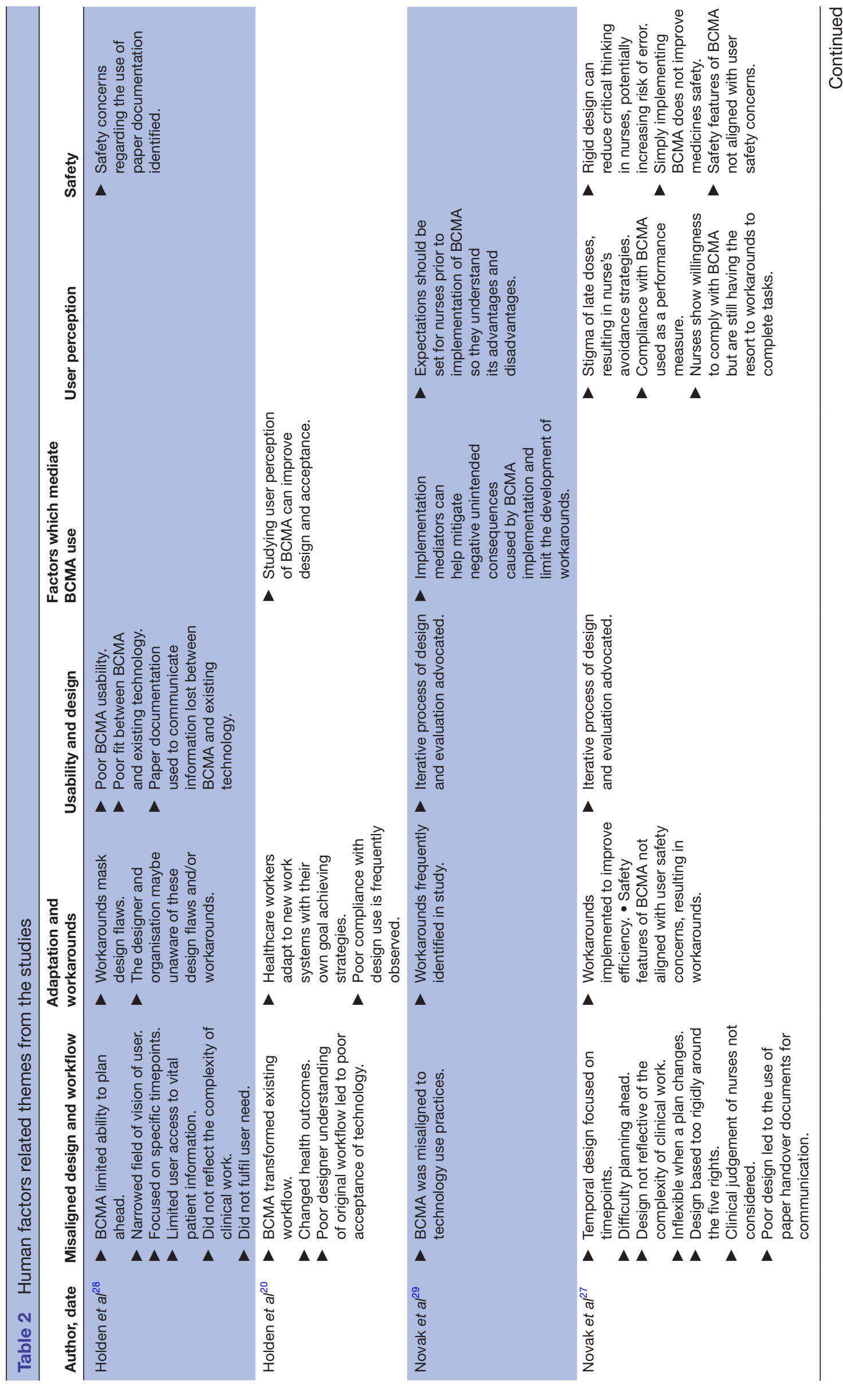




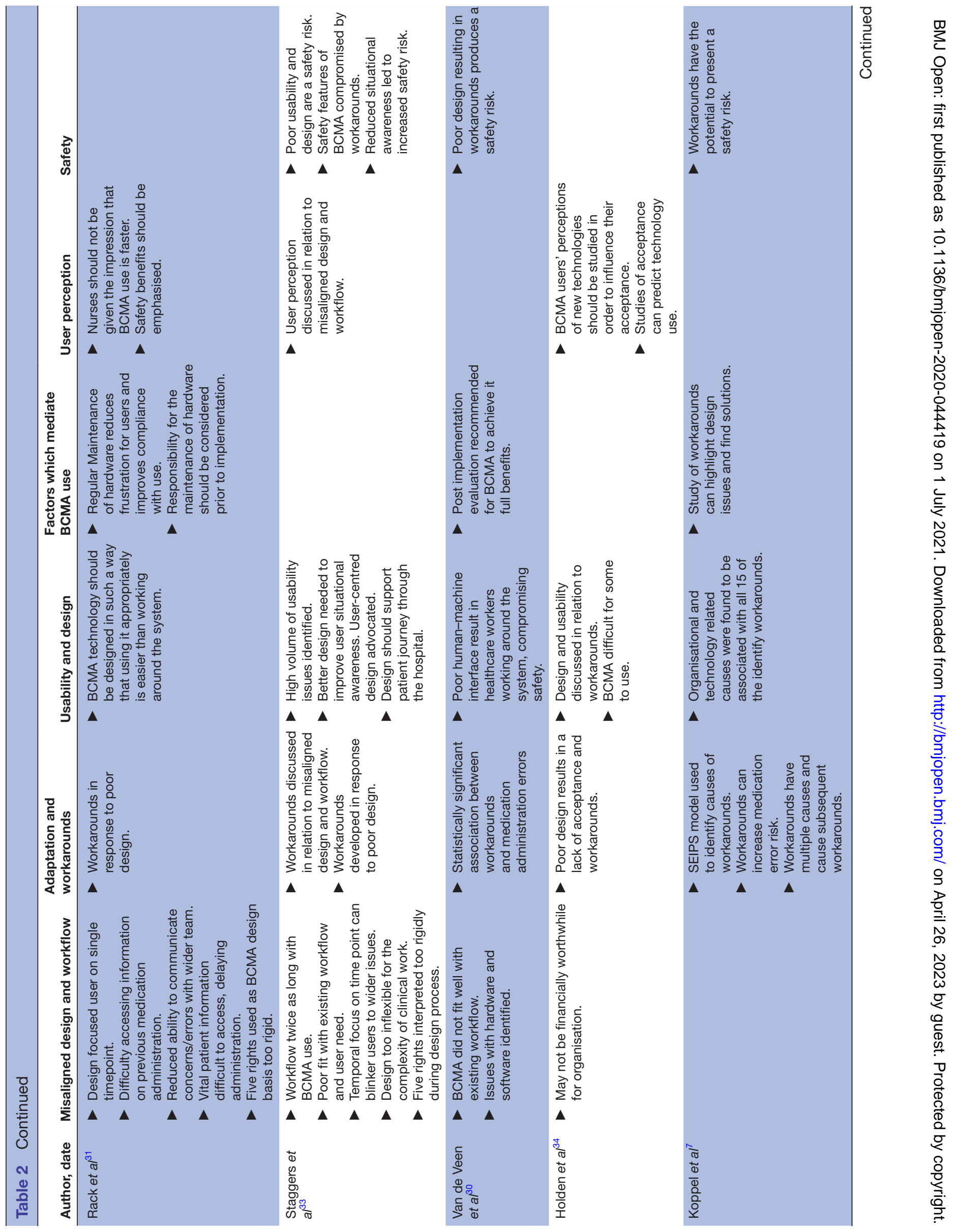




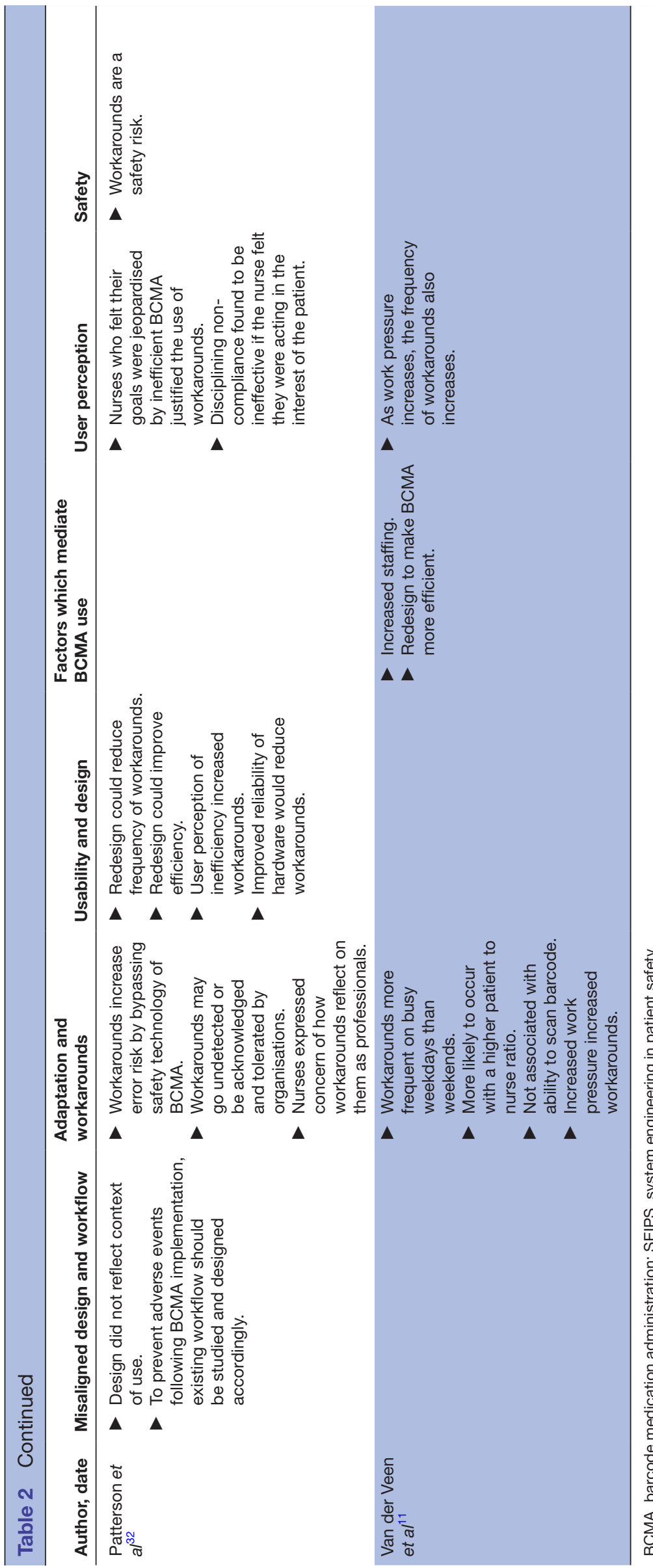


may be unavoidable when introducing technologies that transform workflow. Koppel et al argue that the study of workaround can highlight design flaws in order to remedy them, while Holden et alsuggest that workarounds can be pre-empted and controlled through design. ${ }^{734}$

Koppel et al also posit that workarounds are made more prevalent by poor design. Koppel et al found that workarounds were not only negative but were also sometimes perceived by users as necessary to deliver patient care, finding that consequences of workarounds could be positive, neutral or negative. ${ }^{7}$ Both Koppel et al and Patterson et al advocate human factors approaches to study the causes of workarounds instead of simply introducing policies to increase compliance with intended workflows. ${ }^{732}$

Van der Veen et al s study ${ }^{11}$ examining the factors that contribute to workarounds recommended mandatory nurse to patient ratios, as they found this to be a mediating factor to reduce dangerous workarounds.

\section{Design and usability}

Design and usability issues were identified by most studies as a factor influencing successful BCMA use.

The studies reviewed linked poor design and implementation to increased medication errors and reduced situational awareness. ${ }^{73}$ Patterson et als observational study found that many workarounds could be eliminated by redesign and many of the processes could be made more efficient. ${ }^{32}$ Holden et al argue that usability should be a priority, noting that if the difficulty of use outweighs the benefit, from the user's perspective, workarounds and non-compliance will be more prevalent. ${ }^{28}$ Rack et al argue that the goal of design should be to work in such a way that it is easier to use it correctly than workaround the system to achieve goals. ${ }^{31}$

Many of the papers identified issues with poorly designed hardware and software. Staggers $e t$ al reported frustration and multiple login requests to access the BCMA and eMAR systems studied. Also, the systems could not accommodate patients moving to different areas in the hospital, due to design, which caused confusion regarding whether or not medications had been given. Staggers et al reasoned that better interoperability and patient-centred design could alleviate many of these issues. ${ }^{33}$ Patterson $e t$ $a l$, Koppel et al and Rack et al identified hardware issues such as barcode scanner tethers being too short, workstations on wheels being too bulky to enter treatment rooms and inadequate internet connectivity leading to delays in workflow. ${ }^{73133}$ Van der Veen $e t$ al found that inadequate human-computer interfaces result in frustration and workarounds. ${ }^{30}$

The majority of papers advocated evaluation and re-evaluation during implementation and beyond to take full advantage of safety features and identify the causes of workarounds in order to redesign the system. ${ }^{27} 28$ 30-33 Koppel $e t a l$ and Novak et al advocate ensuring that the designers of the BCMA system understand the current medication administration workflow and environmental and technical factors that may result in poor acceptance and reduce utilisation of new technology. This process should include a pre-implementation assessment to understand user needs and ongoing evaluation, allowing for redesign as issues occur. ${ }^{727}$

\section{Factors which mediate BCMA use}

Many studies identified factors which can ease BCMA implementation, reduce unintended consequences such as workarounds and improve acceptance of new technologies. Factors identified include conducting research that establishes user needs and perceptions of technologies, engaging individuals who act as mediators for both users and designers, ensuring users are aware of system capabilities and limitations and organisational commitment to ensuring hardware is maintained and appropriate for the environment, including sufficient staffing levels.

Holden $e$ t $a l \mathrm{~s}^{20}$ study into user perception and acceptance examined expectations of use pre and post BCMA implementation. Three aspects of medication administration were studied: matching medication to MAR, checking patient ID and documentation. After BCMA implementation, nurses reported decreased likelihood of error, increased likelihood of error detection, increased usefulness, accuracy and consistency for matching medication and identifying the patient. However, they also reported decreased time efficiency and decreased usefulness with regard to documenting actions on the BCMA system. Holden et al suggest that while HITs such as BCMA have a transformative impact on workflow, these changes are measurable and can be mediated by design, if users' expectations and needs are explored prior to development and implementation.

Similarly, when examining how to reduce unintended consequences when switching to a new system such as BCMA, Novak et $a l^{29}$ argued that users' expectations should be set prior to implementation for them to develop an understanding of system capability and limitations. Novak et als study followed a group of mediators who acted as user advocates during BCMA implementation, maintaining timely communication with hospital management and system designers, resulting in a more iterative and evolving implementation process. This style of implementation helped to mitigate negative unintended consequences.

Rack et $a l^{31}$ conducted a survey of 220 nurses using BCMA and held focus groups. Although $90 \%$ of survey respondents agreed that BCMA was safer, many recounted situations where compliance with the BCMA system was not possible, $63 \%$ reported instances of giving medication without scanning the patient, $72 \%$ reported occasions when they did not scan the medication barcode and $40 \%$ reported sometimes scanning medication post administration. Focus groups discussed scenarios where compliance with BCMA was problematic. Overall, 30 scenarios were identified where a workaround was necessary to administer medication. Rack et al emphasises the need to set user expectation prior to BCMA implementation, presenting BCMA as no more time efficient but safer. 
In addition, they note that technology will need maintenance and this needs to be delegated to avoid the frustration of failing or inappropriate equipment. Koppel $e t$ $a l$ also noted that users overestimate the risk elimination ability of BCMA and underestimate the safety features. There is a need for ongoing education to encourage correct use, and for hospital management to thoroughly examine their technological, environmental and social contexts before choosing a BCMA technology. ${ }^{7}$

\section{User perceptions}

Two papers reported that user perception impacted on successful implementation and user compliance. ${ }^{33} 34$ The use of BCMA compliance as a performance measure was found to be unsuccessful and resulted in resistance, particularly where users felt they were acting in the best interests of their patients by employing workarounds. However, users also reported feeling guilt and stigma if they were unable to complete an administration in line with the BCMA system workflow.

Both Novak et $a l^{29}$ and Holden et $a \hat{l}^{84}$ identified a reported stigma regarding late doses and how nurses attempted to avoid this stigma via workarounds. In reanalysing these studies, Novak $e t a l^{29}$ identifies an issue with using BCMA compliance as a performance measure, finding that nurses withholding medication for a legitimate reason were not able to communicate this, resulting in the feeling that they had done something wrong. One hospital punished non-compliance staff and used it as a performance measure, while the other provided continual coaching of staff with the emphasis on safety. Koppel $e t a l^{7}$ suggest that it is not enough to tell staff to comply; rather, a constant evaluation of BCMA use is necessary to improve safety. Holden $e t a l$ 's later study ${ }^{34}$ of nurses' acceptance of BCMA found that nurses already dissatisfied with BCMA are unlikely to use it to its full capacity, only being compliant enough to achieve their goals. Patterson $e t a l^{2}$ also found that policies, sanctions and training were unlikely to improve compliance if users felt that BCMA use jeopardised their ability to provide adequate patient care and achieve their goals. The increased use of workarounds during times of high work pressure reported by Van der Veen et alsuggests that users perceive BCMA as being inefficient, only fully complying with the technology when they have time to do so. ${ }^{11}$

\section{Safety}

The main purpose of BCMA is to improve patient safety; the majority of studies included in this review did not focus on the safety benefits of BCMA but instead used human factors methods to establish the underlying causes of unintended consequences. Nonetheless, there is some evidence that BCMA has this intended effect; for example, Koppel et al analysed 307698 BCMA alerts as well as focused observations, and found over 23000 alerts that appeared to lead to the user changing their action. ${ }^{7}$ However, these studies are unable to conclude that BCMA is safer, instead finding that BCMA has the potential to improve safety. ${ }^{20} 2728$ The issue of improved safety with BCMA technology is complex, and simply having the technology does not make medication administration safer. Increased safety is context dependent, relying on numerous other factors. Rack $e t a l^{11}$ found that the majority of nurses believed BCMA technology was safer but also reported numerous scenarios where they had to bypass the safety features to administer medication.

\section{DISCUSSION}

The aim of this literature review was to identify how human factors influenced the usability and adoption of BCMA use. Studies using a human factors approach revealed a mismatch between BCMA system design and the existing workflow, caused by poor system design, which led to poor user acceptance and the development workarounds which presented a safety risk to patients. A secondary objective was to describe how human factors related determinants for BCMA have been researched and reported by healthcare and human-computer interaction disciplines. However, it became apparent that the studies included could not easily be divided into these two disciplines. Instead, the use of a human factors approach yielded a wide range of narratives, differing time points, outcomes of interest and measures of success. Despite the variety of research focuses, the themes identified were largely complementary and most studies acknowledged how their area of interest was connected to and had consequences for, the overall themes. What does differ is the measures of success in terms of BCMA use. For those studying design, technologies which fit the existing workflow, address clinical demand and improve user situational awareness are considered successful. ${ }^{202728}$ For those researching the safety consequences of workarounds, increased compliance with BCMA use, reduced workarounds and hence safer medication administration are markers of success. ${ }^{71130-32}$ For users, increased efficiency was a priority, ${ }^{34}$ while implementers were concerned with user acceptance and appropriate use of the new BCMA system. ${ }^{29}$ While the measures of success differ, they are all clearly related; the voice missing from this research is that of designers themselves: there is a consensus that system designers do not fully understand user needs and this may be the cause of many of the reported issues; how this is shared with those designing the systems is less clear.

The themes of this review are broadly in line with previous systematic and scoping literature reviews examining BCMA use ${ }^{14} 35$; ; it differs by capturing diverse research focuses and outcomes of interest to represent multiple perspectives. Combined, these provide valuable insights into the successful use of BCMA from numerous actors within the process. The inclusion of human factors highlighted the many different research interests and measures of success regarding BCMA use. Some previous literature reviews focused on particular areas of BCMA use, such as safety or design. ${ }^{35} 36$ Others explored the connection between workarounds and safety, concluding 
that BCMA has the capacity to reduce medication errors if used correctly. ${ }^{14}{ }^{37}$ Voshall $e t a l^{35}$ advocated improved compliance to realise the safety benefits of BCMA, while Hassink et $a l^{36}$ highlighted how system design, workflow mismatch and implementation strategies influence the safety of BCMA but noted that the studies reviewed often did not elaborate on how BCMA was implemented or how the workflow mismatch was addressed. Debono et $a l$ s review ${ }^{14}$ focuses on workarounds and why nurses use them to achieve their goals; they consider the wider context of healthcare delivery and conclude that the nurses' perspective must be understood to reduce workarounds and improve bedside care. More recent studies show that medication related factors, such as the time of the medication round and route of administration, and other factors, such as the barcode integrity, may also influence the likelihood of workarounds. ${ }^{1138}$ By using human factors research to draw on many different voices within BCMA research, this review provides themes across a spectrum of activity for BCMA, from design to adoption.

By reviewing human factors studies which focus on system design, workflow mismatch, informatics and users, it becomes clearer how the identified themes relate to each other. The misalignment in system designed workflow and clinical workflow stems from designers not fully understanding the nature of work in the healthcare setting, as discussed by eight of the selected papers. ${ }^{19} 2627$ 29-31 33 The juxtaposition of complex tasks coupled with changing priorities seems to clash with the rigid, temporally focused BCMA design reported by several studies. ${ }^{27} 283133$ The use of the five rights of medication administration was discussed by Novak et al and Rack et $a l,{ }^{2731}$ suggesting that its use as a guide for BCMA design results in an overly rigid system.

The 'five rights' check list which is designed for use by nurses at the point of medication administration is in practice applied with more flexibility than is acknowledge by BCMA system design. In reality, there are many occasions when a nurse may have to reframe or rationalise one or more of the 'five rights', such as availability of stock, urgency of medication and patient access. ${ }^{28}$ There is an apparent assumption that a formulaic, stepwise BCMA system will lead to increased safety, but healthcare is complex, the ability to adapt to changing situations is essential and inflexible systems may clash with the nature of work ${ }^{39}$ and result in resistance, workarounds and increased safety risks.

Nurses are frequently required to reorganise their work to achieve quality care, often in response to factors beyond their control such as policy, organisational pressure, available technology and demand. ${ }^{27}{ }^{40}$ An important part of the nurse's role is to effectively manage these competing pressures and to advocate for their patients' needs. This review found many examples of problem-solving behaviours in nurses. ${ }^{20}{ }^{27}$ Overly prescriptive design in technology challenges nurses' identity and role. ${ }^{14}$

Policies enforcing compliance with BCMA technology and disciplining non-compliant users was not found to be effective. ${ }^{32}$ The BCMA systems studied frequently reduced perceived efficiency, failed to make essential information available and reduced critical thinking and situational awareness. ${ }^{26} 273031$ Poorly designed BCMA creates additional hurdles to patient care and bypassing the BCMA system could be perceived as justifiable if it is in the interests of the patient. ${ }^{33}$ However, the resulting workarounds circumvent the safety features of BCMA and expose the patient to increased risk of medication error. This conflict was evident in the literature reviewed: nurses agreed that BCMA use was safer but frequently encountered scenarios where they could not complete a task and use the BCMA technology correctly. ${ }^{31}$ Conversely, users can sometimes overestimate the risk reduction capability of BCMA, relying on the technology to identify an error rather than a combination of the technology and their own clinical judgement. ${ }^{31}$

Workarounds were witnessed in every observational study in the review, but the terminology used to describe them differed: from adaptive and problem-solving behaviours to deviations and errors. ${ }^{27} 30$ The use of different terminology surrounding workarounds implies either negative or positive attitudes towards them. ${ }^{14}$ In the studies presented, safety focused papers often examined workarounds as an adverse event risk, while design and usability focused papers often described them as unavoidable and even informative. ${ }^{28}$ Many of the papers were divided on the consequence of workarounds. ${ }^{9}$ While the association between workarounds and medication errors is concerning, most studies acknowledge that workarounds are unavoidable when introducing a transformative technology into an existing workflow, and it is poor design and implementation that make them problematic. $^{730}$

Studies included in this review agree that many of the problems with BCMA use are rooted in designers not fully understanding the complexity of clinical work. Measures to manage these design mismatches include careful and long-term implementation strategies, organisational and technological structures which encourage correct BCMA use and close monitoring of workarounds. However, many of these strategies seem to be compensating for less than adequate design; how to redesign systems to better match clinical need is not really addressed and the designer perspective is absent from the studies reviewed. However, the differing findings and perspectives act as a powerful message that there is a greater need for close working throughout design and deployment for BCMA to achieve its recognised potential in improving patient safety.

\section{Implications for clinicians and policy-makers}

The literature identified many mediating factors and potential strategies for enhancing BCMA use for clinicians, policy-makers and users. An understanding of users' perceptions of a new technology prior to implementation can be predictive of overall acceptance and can guide design. ${ }^{20}$ Employing staff who are trained to act as mediators to ease implementation and act as 
a bridge between users and designers was found to be helpful by Novak et $a .^{29}$ Ensuring that software and hardware are appropriate for the environment and properly maintained to reduce frustration and mistrust in technology, along with appropriate staffing levels, require an organisational commitment and cannot be achieved by an individual nurse. ${ }^{11}{ }^{31}$ Most studies recommended pre implementation evaluation and constant re-evaluation during the implementation phase with human factors frameworks to identify the causes of poor compliance with technology and inform redesign of the BCMA system. Success is dependent on collaboration between designers, informatics experts, users and the organisation to prevent workarounds persisting and becoming risks to safety. It may be necessary to view BCMA (and other HIT) system vendors as long-term partners, establishing a good understanding of user needs, organisational capability and how usability issues will be addressed following implementation.

\section{Recommendations for further research}

As noted above, the designers of BCMA systems are rarely visible in the discourse around their implementation and use. Studies of workarounds tend not to question the details of specific BCMA design, but to focus more on the complexity of the broader system. Further research is needed to better understand how new technologies can be designed and safely implemented into complex healthcare settings. This review, along with others, ${ }^{14} 3536$ has made it clear that BCMA technology is a component within a complex system of medication administration. Further interdisciplinary research is needed to better understand how technology to support safer medication administration can be designed to accommodate the complexities of use while also supporting staff in managing that complexity. In parallel, it is important to improve both user experience and patient safety. Future research should also examine the long-term effects of BCMA, not just at the point of implementation but as use evolves over years, to evaluate whether its safety benefits are sustainable as the environment and users change.

\section{Limitations and strengths}

Most studies included in this review were small in sample size and conducted in the USA. They relied on qualitative research methodologies such as observation, focus groups and surveys. Many of the studies triangulated their qualitative findings with quantitative data, such as BCMA compliance reports, to better understand what was being observed in practice and to make their findings more generalisable.

As this study particularly examined BCMA implementation with a human factors lens, many BCMA studies were excluded, resulting in only 11 papers being included in the final review. This has given a focused view of the available research including evidence from both healthcare and human-computer interaction perspectives.
The search strategy of this review was independently repeated by a second reviewer to reduce the risk of bias, and a good level of agreement was achieved.

\section{CONCLUSION}

This review found that successful BCMA use is eased by a clear understanding of existing workflow and user needs. Evaluation of BCMA technology pre, during and postimplementation can help to identify workarounds and guide redesign, organisational commitment to understanding and resolving issues with BCMA acceptance and collaboration between users and system designers. Human factors principles can be used to understand causes of poor BCMA use and acceptance in the complex healthcare setting, and can unify the voices and experiences of those using the technology. This should not just enable people to compensate for poor design but also guide system designers to improve system design and therefore patient safety.

Acknowledgements RW completed this work during her UCLH NHS Foundation Trust CEO and UCLH-UCL CMORE clinical research fellowship.

Contributors RW: Protocol design, literature review development, literature search, analysis and manuscript writing. RA: Independent second literature search, review of themes and manuscript review. AB: Protocol guidance, review and guidance on search strategy, identified themes and manuscript review and finalising. $\mathrm{YJ}$ : Protocol guidance, review and guidance on search strategy, identified themes and manuscript review and finalising.

Funding The authors have not declared a specific grant for this research from any funding agency in the public, commercial or not-for-profit sectors.

Competing interests None declared.

Patient consent for publication Not required.

Provenance and peer review Not commissioned; externally peer reviewed.

Data availability statement All data relevant to the study are included in the article or uploaded as supplementary information.

Supplemental material This content has been supplied by the author(s). It has not been vetted by BMJ Publishing Group Limited (BMJ) and may not have been peer-reviewed. Any opinions or recommendations discussed are solely those of the author(s) and are not endorsed by BMJ. BMJ disclaims all liability and responsibility arising from any reliance placed on the content. Where the content includes any translated material, BMJ does not warrant the accuracy and reliability of the translations (including but not limited to local regulations, clinical guidelines, terminology, drug names and drug dosages), and is not responsible for any error and/or omissions arising from translation and adaptation or otherwise.

Open access This is an open access article distributed in accordance with the Creative Commons Attribution Non Commercial (CC BY-NC 4.0) license, which permits others to distribute, remix, adapt, build upon this work non-commercially, and license their derivative works on different terms, provided the original work is properly cited, appropriate credit is given, any changes made indicated, and the use is non-commercial. See: http://creativecommons.org/licenses/by-nc/4.0/.

ORCID iDs

Rachel Williams http://orcid.org/0000-0002-8497-6945

Ann Blandford http://orcid.org/0000-0002-3198-7122

Yogini Jani http://orcid.org/0000-0001-5927-5429

\section{REFERENCES}

1 Hutton K, Ding Q, Wellman G. The effects of bar-coding technology on medication errors: a systematic literature review. J Patient Saf 2021;17:e192-206. 
2 Institute of Medicine. To Err Is Human: Building a Safer Health System [Internet], 1999. Available: https://www.nap.edu/catalog/ 9728/to-err-is-human-building-a-safer-health-system

3 Department of Health. Medication errors: short life working group report [Internet]. GOV.UK, 2018. Available: https://www.gov.uk/ government/publications/medication-errors-short-life-workinggroup-report

4 Elliott RA, Camacho E, Campbell F. Prevalence and economic burden of medication errors in the NHS in England 2018;174.

5 WHO. World Health Organization. The third WHO global patient safety challenge: medication without harm, 2020. http://www.who.int/ patientsafety/medication-safety/en/

6 Aspden P, Wolcott J, Lyle Bootman J. Preventing Medication Errors: Quality Chasm Series | IHI - Institute for Healthcare Improvement [Internet], 2006. Available: http://www.ihi.org:80/resources/Pages/ Publications/PreventingMedicationErrorsQualityChasmSeries.aspx

7 Koppel R, Wetterneck T, Telles JL, et al. Workarounds to barcode medication administration systems: their occurrences, causes, and threats to patient safety. J Am Med Inform Assoc 2008;15:408-23.

8 Bates DW, Cullen DJ, Laird N, et al. Incidence of adverse drug events and potential adverse drug events. Implications for prevention. ADE prevention Study Group. JAMA 1995;274:29-34.

9 Kopp BJ, Erstad BL, Allen ME, et al. Medication errors and adverse drug events in an intensive care unit: direct observation approach for detection. Crit Care Med 2006;34:415-25.

10 Leape LL, Bates DW, Cullen DJ, et al. Systems analysis of adverse drug events. ade prevention Study Group. JAMA 1995;274:35-43.

11 van der Veen $\mathrm{W}$, Taxis $\mathrm{K}$, Wouters $\mathrm{H}$, et al. Factors associated with workarounds in barcode-assisted medication administration in hospitals. J Clin Nurs 2020;29:2239-2250.

12 Poon EG, Keohane CA, Yoon CS, et al. Effect of bar-code technology on the safety of medication administration. N Engl J Med 2010;362:1698-707.

13 Bates DW. Using information technology to reduce rates of medication errors in hospitals. BMJ 2000;320:788-91.

14 Debono DS, Greenfield D, Travaglia JF, et al. Nurses' workarounds in acute healthcare settings: a scoping review. BMC Health Serv Res 2013;13:175.

15 Shah K, Lo C, Babich M, et al. Bar code medication administration technology: a systematic review of impact on patient safety when used with computerized prescriber order entry and automated dispensing devices. Can J Hosp Pharm 2016;69:394-402.

16 Section of Pharmacy Informatics and Technology, American Society of Health-System Pharmacists. ASHP statement on bar-codeenabled medication administration technology. Am J Health Syst Pharm 2009;66:588-90.

17 Barakat S, Franklin BD. An evaluation of the impact of barcode patient and medication scanning on nursing workflow at a UK teaching hospital. Pharmacy 2020;8:148.

18 Reason J. Human error. Cambridge University Press, 1990: 324 p.

19 Diamond CC, Shirky C. Health information technology: a few years of magical thinking? Health Aff 2008;27:w383-90.

20 Holden RJ, Brown RL, Alper SJ, et al. That's nice, but what does it do? Evaluating the impact of bar coded medication administration by measuring changes in the process of care. Int $J$ Ind Ergon 2011;41:370-9.

21 Ammenwerth E, Iller C, Mahler C. IT-adoption and the interaction of task, technology and individuals: a fit framework and a case study. BMC Med Inform Decis Mak 2006;6:3.

22 Holden RJ, Karsh B-T. A theoretical model of health information technology usage behaviour with implications for patient safety. Behav Inf Technol 2009;28:21-38.
23 Karsh B-T, Holden R, Escoto K, et al. Do Beliefs About Hospital Technologies Predict Nurses' Perceptions of Quality of Care? A Study of Task-Technology Fit in Two Pediatric Hospitals. Int J Hum Comput Interact 2009;25:374-89.

24 Holden RJ, Carayon P, Gurses AP, et al. SEIPS 2.0: a human factors framework for studying and improving the work of healthcare professionals and patients. Ergonomics 2013;56:1669-86.

25 Carayon P, Schoofs Hundt A, Karsh B-T, et al. Work system design for patient safety: the SEIPS model. Qual Saf Health Care 2006;15 Suppl 1:i50-8.

26 Moher D, Liberati A, Tetzlaff J, et al. Preferred reporting items for systematic reviews and meta-analyses: the PRISMA statement. BMJ 2009;339:b2535.

27 Novak LL, Holden RJ, Anders SH, et al. Using a sociotechnical framework to understand adaptations in health IT implementation. Int $J$ Med Inform 2013;82:e331-44.

28 Holden RJ, Rivera-Rodriguez AJ, Faye $\mathrm{H}$, et al. Automation and adaptation: nurses' problem-solving behavior following the implementation of bar-coded medication administration technology. Cogn Tech Work 2013;15:283-96.

29 Novak LL, Anders S, Gadd CS, et al. Mediation of adoption and use: a key strategy for mitigating unintended consequences of health it implementation. J Am Med Inform Assoc 2012;19:1043-9.

30 van der Veen $\mathrm{W}$, van den Bemt PMLA, Wouters $\mathrm{H}$, et al. Association between workarounds and medication administration errors in barcode-assisted medication administration in hospitals. J Am Med Inform Assoc 2018;25:385-92.

31 Rack LL, Dudjak LA, Wolf GA. Study of nurse workarounds in a hospital using bar code medication administration system. J Nurs Care Qual 2012;27:232-9.

32 Patterson ES, Rogers ML, Chapman RJ, et al. Compliance with intended use of bar code medication administration in acute and long-term care: an observational study. Hum Factors 2006;48:15-22.

33 Staggers N, Iribarren S, Guo J-W, et al. Evaluation of a BCMA's electronic medication administration record. West $J$ Nurs Res 2015;37:899-921.

34 Holden RJ, Brown RL, Scanlon MC, et al. Modeling nurses' acceptance of bar coded medication administration technology at a pediatric hospital. J Am Med Inform Assoc 2012;19:1050-8.

35 Voshall B, Piscotty R, Lawrence J, et al. Barcode medication administration work-arounds: a systematic review and implications for nurse executives. J Nurs Adm 2013;43:530-5.

36 Hassink JJM, Jansen MMPM, Helmons PJ. Effects of bar codeassisted medication administration (BCMA) on frequency, type and severity of medication administration errors: a review of the literature. Eur J Hosp Pharm 2012;19:489-94.

37 Patterson ES. Workarounds to intended use of health information technology: a narrative review of the human factors engineering literature. Hum Factors 2018;60:281-92.

38 Othman EH, Darawad MW. Nurses' compliance with barcode medication administration technology: results of direct observation of Jordanian nurses' practice. Comput Inform Nurs 2020;38:256-62.

39 Hong JY, Ivory $\mathrm{CH}$, VanHouten $\mathrm{CB}$, et al. Disappearing expertise in clinical automation: barcode medication administration and nurse autonomy. J Am Med Inform Assoc 2021;28:232-8.

40 Vos J, Franklin BD, Chumbley G, et al. Nurses as a source of systemlevel resilience: secondary analysis of qualitative data from a study of intravenous infusion safety in English hospitals. Int J Nurs Stud 2020;102:103468. 